\title{
A self-contained microcomputer system for psychological testing
}

\author{
JAMES H. JOHNSON, RONALD A. GIANNETTI, and THOMAS A. WILLIAMS \\ Eastern Virginia Medical School, Norfolk, Virginia 23501
}

\begin{abstract}
This paper describes a newly available microcomputer system for the on-line administration, scoring, and interpretation of psychological tests. User response to the system is reported. Results of a validity study of the system's Minnesota Multiphasic Personality Inventory interpretative program are presented.
\end{abstract}

Computers were first used experimentally for scoring and interpreting psychological tests during the early 1960s (c.f. Pearson, Swenson, Rome, Mataya, \& Brannick, 1965; Piotrowski, 1964). Shortly afterward, computerized test interpretation was made available commercially (Fowler, in press). To use the commercial systems, test answers are recorded on computer-readable media, mailed to the test center for processing, and results mailed back to the clinician. While this service has been an aid to clinicians in many diverse settings throughout the country, it has at least one important drawback. There is often a delay of up to 2 weeks between the time the patient actually takes the test and the time results are returned to the clinician. This response impediment limits the usefulness of such commercial services in many clinical settings.

These relatively awkward procedures were dictated by the constraints and limitations of the technology available when automated test-interpretation procedures were first instituted. Computer equipment with sufficient capabilities to interpret psychological tests was expensive, too expensive for most clinical facilities. Interpretative programs for tests such as the Minnesota Multiphasic Personality Inventory (MMPI) often require more than $128 \mathrm{~K}$ bytes of memory along with directaccess storage media. Such requirements have necessitated the scoring and interpretation of tests on systems that are primarily justified for other applications or for multiple users.

Even the advent of minicomputer technology did not change this situation markedly. Costs of required equipment capabilities were still too great for most institutions. However, this position has changed with the recent introduction of microcomputer technology. It is now possible for individual institutions to afford their own computer systems for the administration, scoring, and interpretation of psychological tests.

Recently, a new commercial service has taken advantage of microcomputer technology to provide

Requests for reprints should be sent to James H. Johnson, Department of Psychiatry and Behavioral Sciences, Eastern Virginia Medical School, P.O. Box 1980, Norfolk, Virginia 23501. inexpensive automated systems for the on-line administration and interpretation of psychological tests. The Lab-II, manufactured by Psych Systems, ${ }^{1}$ is a self-contained, on-line, microcomputer-based psychological testing station located at the user's site that is programmed to administer, score, and interpret the Strong-Campbell Vocational Interest Inventory, the Shipley-Hartford Intelligence scale, the MMPI, measures of arithmetic and memory ability, Beck Depression and Hopelessness, and an index of test dissimulation. The Lab-II has an advantage over previous commercial systems in that automated test interpretations are available to the clinician immediately following administration of testing. Furthermore, the vendor claims that test reports are specifically designed for real-time use in clinical settings and that interpretative programs follow established scientific conventions. For example, the MMPI interpretation program follows the procedures outlined by Miller, Johnson, Klingler, Williams, and Giannetti (1977). In addition, the Lab-II can be programmed by the user for other purposes, such as management information and billing.

The Lab-II is packaged in an integrated cabinet that measures only $13.5 \mathrm{in}$. high and rests conveniently on a standard typewriter stand. Within this package are two Z80-A microprocessors (the second processor is used as a disk controller), two soft-sector mini-floppy diskettes with a capacity of $180 \mathrm{~K}$ bytes, $32 \mathrm{~K}$ bytes of dynamic RAM, a $2.2 \mathrm{~K}$-character/sec electroresistive printer, a full keyboard, and a $6 \times 9$ in. cathode-ray tube (CRT) display. Both microprocessors have access to systems memory using DMA techniques. The disk controller follows commands presented through a mailbox in systems memory. After performance of a command function, it interrupts the systems processor and informs it of status information using a mailbox approach. The DMA controller is also used to interact with system memory to pass screen-image information to line buffers in the display system. Line-buffered data are processed through a character generator for presentation on the CRT.

The operating system for the Lab-II is relatively sophisticated when compared with others available 
on 8-bit processors. It performs such tasks as file management, buffering, record blocking and unblocking, directory maintenance, searching, indexing, automatic error retry, program loading, and program chaining. User programs are completed in commercial BASIC Plus, a superset of Dartmouth BASIC.

Because it seemed to us that the Lab-II had potential for wide applicability in mental health service delivery settings, we decided to evaluate this system. The results of that evaluation are presented below. In the first section, anecdotal information about programmable use of the system is noted. A formal evaluation study of the MMPI test interpretation system is presented in the second section.

\section{USER PROGRAMMING}

We have now completed nearly two dozen programs on the Lab-II and have found the system easy to use. Operation is quite simple (e.g., systems initialization is automatic). The BASIC programming system is comprehensive and yet easy to use for most problems.

However, certain difficulties are evident for more complex problems. Files cannot be created in BASIC. They are created using separate file operators after exiting from BASIC. Once the file is cleared, the BASIC interpreter is reloaded into the system for further processing.

Another important programming problem that we became aware of relates to the fact that the BASIC interpreter does not include a COMMON statement. While this is not a severe problem, it is relatively troublesome. The standard Lab-II system has $5 \mathrm{~K}$ bytes of memory allocated for user programming. Because this amount of space is small, many programs need to be chained. Lack of a COMMON statement means that each subprogram within a chain must include its own variable list.

\section{THE MMPI INTERPRETATION SYSTEM}

Very little validity information is available regarding the interpretative approaches used by most commerical test scoring and interpretation services. This is especially true for the MMPI (Butcher, 1977). For this reason, we undertook a brief study of the validity of the Lab-II automated MMPI interpretation program.

Twenty-five patients (14 females, 11 males) from the Norfolk Community Mental Health Center were tested on the Lab system. The MMPI profile and computer-generated interpretation were mailed to two independent, experienced clinical psychologists located in another city. These clinicians were asked to review each profile blindly and compare their interpretation with the interpretation generated by the Lab system. The same clinicians were asked for two separate ratings:
"How good is the agreement of the automated interpretation with your clinical interpretation, given that you were asked to interpret the same profile blindly?" and What is the adequacy of the automated narrative in terms of being inclusive, and yet succinct?" Ratings for each question were on an anchored 5-point scale ( 1 = unsatisfactory, $2=$ poor, $3=$ average, $4=$ good, $5=$ excellent $)$.

Agreement between the two raters was moderate. On the first question, interrater reliability equaled .38; on the second question reliability was .20 . However, the separate mean ratings were relatively consistent.

In answer to the question about the clinician's agreement with the automated interpretation, the first clinician rated the interpretation as "good" (mean $=3.96, \mathrm{SD}=1.06$ ) and the second clinician rated agreement as "average" (mean $=3.00, \mathrm{SD}=1.50$ ). In response to the question of the adequacy of the computer narrative, the first clinician rated results in the "average" to "good" range (mean $=3.80, \mathrm{SD}=1.12$ ); the second clinician also rated results in the "average" to "good" range (mean $=3.20, \mathrm{SD}=1.47$ ). These findings suggest that the Lab system's automated MMPI interpretive report is seen by clinicians as at least equal to those that they complete.

\section{DISCUSSION}

Our experience suggests that the Lab-II psychological testing computer system has utility for general-purpose computing problems. Furthermore, the MMPI scoring and interpretation system results in interpretations that clinicians consider to be similar to those they complete.

The Lab-II represents the first commercially available system to make use of microcomputer technology in mental health care delivery settings. Because of this technology, it is now possible to locate a self-contained computer system in a clinical setting in order to obtain computerized test interpretations on a real-time basis at an affordable cost. This kind of system symbolizes the power of the microcomputer and its future potential for impacting the behavioral sciences.

\section{REFERENCES}

Butcher, J. N. The MMPI. In O. K. Buros (Ed.), The eighth mental measurements yearbook. Highland Park, N.J: Gryphon Press, 1977.

Fowler, R. D. The automated MMPI. In J. B. Sidowski, J. H. Johnson, \& T. A. Williams (Eds.), Technology in mental health care delivery systems. Norwood, N.J: Ablex Publishing, in press.

Mirler, D. A., Johnson, J. H., Klingler, D. E., Williams, T. A., \& Giannetti, R. A. Design of an on-line computerized system for MMPI interpretation. Behavior Research Methods \& Instrumentation, 1977, 9, 117-122.

Pearson, J. S., Swenson, W. M., Rome, H. P., Mataya, P., \& Brannick, T. L. Development of a computer system 
for scoring and interpretation of Minnesota Multiphasic Personality Inventories in a medical clinic. Annals of the New York Academy of Sciences, 1965, 126, 682-692.

Plotrowski. Z. A. Digital-computer interpretation of inkblot test data. Psychiatric Quarterly, 1964, 38, 1-26.

\section{NOTE}

1. Psych Systems is located at Suite 206, Medical Tower, Norfolk, Virginia 23507.

(Received for publication June 29, 1978.) 